\title{
Depressive reactions to lung cancer are common and often followed by a poor outcome
}

\author{
G. Buccheri
}

Depressive reactions to lung cancer are common and often followed by a poor outcome. G. Buccheri. (OERS Journals Ltd 1998.

ABSTRACT: In diverse human malignancies, including lung cancer, quality of life (QOL) affects the clinical outcome of patients. Many QOL dimensions, however, are influenced by physical status. The current study was undertaken to delineate the role of depression, a state of mind not necessarily influenced by somatic factors.

All patients, seen for a newly diagnosed carcinoma of the lung, received a copy of the Self-rating Depression Scale (SDS) proposed by W.W.K. Zung. One hundred and thirty three patients were able and willing to complete the questionnaire. A depression index was calculated, as originally described. Nineteen other prognostically important variables were recorded and available for evaluation.

Among the 95 patients who completed all 20 items included in the questionnaire, 53 had an SDS index $ð 50$ (no depression), while 42 were moderately or severely depressed (SDS index $>50$ ). The survival of depressed patients was significantly lower $(p=0.048)$. Diverse SDS subscales were associated with survival, in either univariate or multivariate analysis. SDS item 19, "I feel that others would be better off if I were dead", emerged as the most significant one. A multivariate model of survival, constructed using only SDS data, had a global Chi-squared value of 29.78, and a p-value of $\mathbf{0 . 0 0 0 2 3}$.

Based on this evidence, it appears that a relationship does exist between patients' depression and prognosis, at least in lung cancer. Further studies are needed to clarify the prognostic effect of depression in more specific (and homogeneous) subgroups of patients.

Eur Respir J 1998; 11: 173-178.

The evaluation of quality of life (QOL) has become part of the overall management of patients with cancer. This is particularly true when survival is likely to be short and treatments are expected to be toxic and only moderately active. Lung cancer (LC) is very common, and predominantly affects the elderly population. Tolerance to aggressive cancer treatments is poor, and such treatments are largely ineffective [1]. Obviously, the QOL of such patients merits the greatest attention.

In recent years, diverse reports dealing with the assessment of QOL in LC have been published [2-6], and an array of different methods described [7-9]. One of the most intriguing applications of self-rated QOL scores is their use as prognostic factors. In an early Scandinavian study [2], psychosocial wellbeing and general symptoms were found among the factors that significantly predicted survival of patients with advanced non-small cell lung cancer (NSCLC). In a later report by GANZ et al. [4], the supposed relationship was hidden by the small number of patients studied (only 40 in total). In the same year, however, members of the Lung Cancer Study Group, analysing the charts of 178 patients from four phase III trials, confirmed the prognostic importance of QOL [5]. More recently, using a new instrument of assessment [9], BucCHERI [6] confirmed the supposed relationship between QOL and prognosis.
QOL is a multidimensional concept with a particularly wide meaning. It includes, at least, a rating of the physical, psychological, and functional status with, where resources permit, an evaluation of the social functioning [10]. Most QOL dimensions are influenced by the physical status of patients. Physical scores are affected by the seriousness of the disease, and many functional, social, and psychological responses may be affected as well. The prognostic value of QOL might be a simple reflection of the severity of the illness. The question remains whether those aspects of QOL that are less correlated with the physical wellbeing, are still prognostically important.

To the author's knowledge, this is the first study to assess depression and its prognostic significance in patients with lung cancer.

\section{Patients and methods}

\section{Patients}

All patients, seen between June 1994 and January 1996 for a new pathologically proven bronchogenic carcinoma at a specialist tertiary referral centre (the A. Carle Hospital of Chest Diseases), were considered for this study. Exceptions were made for those individuals with a very poor 
performance status (Eastern Co-operative Oncology Group (ECOG) scale $>3$ [11]), since it was felt that psychological assessment might prove too difficult to perform in those conditions, and also for patients with severely impaired intellectual function. Using a personalized approach which took into consideration the patient's social, cultural, and emotional status, two doctors (G.B. and D.F.) provided the necessary information concerning diagnosis, prognosis, and treatment, along with an explanation of the aim and the characteristics of the study. Then, a written consent for the study was requested, in accordance with the guidelines of the institutional review board. Any patients expressing concern about their full understanding of the questionnaire, were instructed to seek directions from their relatives or a nurse on how to complete it.

The mandatory clinical work-up evaluation included: history; physical examination, assessment of performance status (PS) and loss of body weight (during the 6 months preceding the diagnosis); complete blood cell counts; serum biochemistry; bronchoscopy; chest radiographs; and computed tomography of thorax, brain, and upper abdomen. A new staging procedure, consisting of Indium-111 anti-carcinoembryonic antigen (CEA) monoclonal antibody scintigraphy [12], was added to the evaluation of all surgical candidates. Cell types and tumour stages were classified according to internationally adopted criteria $[13,14]$.

\section{QOL assessment}

A nurse gave each patient four different QOL questionnaires and showed how to complete them. This report deals with depression, which is a marginal aspect of the QOL evaluation. To assess the desired feature, the Self-rating Depression Scale (SDS), as described by ZuNG [15], was administered along with three other questionnaires [16]. All cards were collected during the next 2-3 days spent in hospital, or soon after the administration, in the case of an out-patient visit. Nurses collected and reviewed the cards and, when reports were found incomplete or not sufficiently reliable, offered assistance to patients, so that the quality of the reports could be improved. A few patients expressed embarrassment in responding to personal questions. In such cases, the nurses were instructed to accept incomplete SDS cards, unless missing responses were more than four, i.e. $20 \%$ of the card (more incomplete questionnaires were disregarded). The clinical characteristics of patients who were unable or unwilling to complete satisfactorily the SDS questionnaire, were recorded and used for later comparisons.

A detailed description encompassing feasibility, reliability, and validity of the Zung scale was published, several years ago, by Zung $[15,17,18]$. This scale, originally proposed in English, has been translated and available for use in 10 languages, including Italian [17]. It contains 20 categorical self-assessment scales covering: pervasive effect; physiological equivalents or concomitants; and psychological concomitants. Ten items are worded symptomatically positive, and 10 symptomatically negative. In scoring items, a value of $1,2,3$ or 4 is assigned depending upon whether the item was worded positively or negatively. Nondepressed patients have a low score on the scale and a low index (sum of raw scores divided by 80 , the maximum score); the opposite occurs for more depressed patients.

\section{Statistical analysis}

Descriptive statistics were used to characterize the sample. The Student's t-tests, Pearson Chi-squared tests, and Mann-Whitney U-tests [19] were employed, as appropriate, to analyse differences between patients allocated in different subgroups. The length of survival was calculated from the distribution of the SDS questionnaire to the patient's death or to July 1996. Few patients abandoned their follow-up programme; though when this occurred, the status of alive or dead was ascertained by means of a telephone interview with the same patient, the family, the house doctor, or the municipal registry office. Univariate analyses of survival were based on the Kaplan-Meier product-limit estimates of the distribution [20]. For each of the 20 items forming the SDS questionnaire, survival analyses were accomplished using both the original four scale categorizations and other categories derived by the amalgamation of the original four classes into the two or three strata with similar numerical weight. Multiple survival curves were compared using the algorithms developed by LeE and Desu [21], an extension of the Gehan's generalized Wilcoxon test [22]. The Gehan's test was used to compare two sample survivals. The relative weight of each prognostic factor was estimated using Cox's proportional hazards regression analysis and the statistical significance calculated by means of the maximum likelihood ratio [23]. To select the simplest and most predictive multivariate model, a stepwise backward selection procedure was followed with a $\mathrm{p}$-value of 0.1 set to enter, and a $\mathrm{p}$ value of 0.15 set to remove variables. A p-value of less than 0.05 was considered statistically significant in the analysis of the SDS index, as well as for any multivariate regression. For the 20 items of the Zung scale individually considered' the Bonferroni correction was used and a critical p-value of $<0.0025$ requested. All tests were twosided. Data were processed using the STATISTICA for Windows package (release 4.5, StatSoft Inc., Tulsa, OK, USA, (C1993).

\section{Results}

\section{Baseline characteristics of patients}

In total, 167 newly diagnosed LC patients were seen during the recruitment period. One hundred and fifty four were eligible for this study: 21 refused or were unable to complete at least 16 of the 20 items forming the SDS instrument. The remaining 133 were entered into the study. Comparing the two groups of patients (i.e. registered versus nonregistered patients) no significant difference emerged, except for the complete absence of squamous cell types within the group of the 21 nonregistered individuals $(\mathrm{p}<$ 0.0001, Chi-squared test).

Patients participating in the study had an age and sex distribution typical for the disease. Most of them suffered from cancers of the squamous cell type, a rare occurrence in the Western world, but still quite a typical feature in the rural and mostly unpolluted area in which this study was undertaken $[24,25]$. On average, patients had a low degree of education, being mostly farmers and housebuilding workers. Both the very early and the advanced 
stages of diseases were represented. Performance status was often good or fair. The SDS questionnaire was fully scored by 95 patients; the other 38 patients omitted one or more response. In 53 of these 95 fully compliant subjects, the calculated SDS index was $\partial 50$ (normality range); all the remaining 42 subjects had indexes compatible with mild-to-severe depression. Depressed patients were often older, and their performance status and stage of disease were worse, on average, than that seen in nondepressed patients; however, no difference was statistically significant. The most important clinical and demographic char- acteristics of the cohort studied and their distribution among depressed and nondepressed patients are summarized in table 1. Table 2 summarizes the SDS responses of the whole population.

\section{Survival analysis}

In univariate analyses, higher scores on the scales were often associated with shorter survivals (analyses of the original four-point scales). The trend approached statistical significance in eight circumstances (SDS items 2, 5, 7,

Table 1. - Clinical and demographic characteristics of the cohort studied

\begin{tabular}{lccc}
\hline Demographic and clinical characteristics & Total & Nondepressed & Depressed \\
\hline Patients $\mathrm{n}$ & 133 & 53 & 42 \\
Sex male/female & $122 / 11$ & $51 / 2$ & $38 / 4$ \\
Age yrs & $65 \pm 10$ & $61 \pm 10$ & $69 \pm 9$ \\
Education EL/MS/HS/U & $44 / 61 / 25 / 3$ & $17 / 24 / 10 / 2$ & $14 / 17 / 10 / 1$ \\
Help in SDS response yes/no & $59 / 74$ & $26 / 27$ & $17 / 25$ \\
ECOG-PS 0/1/2/3 & $18 / 65 / 37 / 13$ & $11 / 27 / 11 / 2 / 2$ & $3 / 20 / 15 / 3 / 1$ \\
Histology E/A/S/L/O & $58 / 28 / 11 / 4 / 32$ & $25 / 10 / 6 / 2 / 10$ & $17 / 11 / 2 / 2 / 10$ \\
Stage of disease 1/2/3a/3b/4 & $26 / 6 / 19 / 37 / 45$ & $13 / 4 / 6 / 12 / 18$ & $6 / 0 / 7 / 13 / 16$ \\
T factor 1/2/3/4 & $21 / 48 / 19 / 45$ & $7 / 24 / 7 / 15$ & $5 / 14 / 6 / 17$ \\
N factor 0/1/2/3 & $50 / 12 / 45 / 26$ & $20 / 8 / 16 / 9$ & $15 / 2 / 15 / 10$ \\
M factor 0/1 & $88 / 45$ & $35 / 18$ & $26 / 16$ \\
Serum haemoglobin $\mathrm{g} \cdot \mathrm{dL}-1$ & $13.9 \pm 1.5$ & $14.1 \pm 1.5$ & $13.7 \pm 1.4$ \\
WBC count $\mathrm{n} \cdot \mathrm{m}^{-3}$ & $9347 \pm 3713$ & $9268 \pm 2610$ & $9617 \pm 5054$ \\
& $(\mathrm{n}=132)$ & $(\mathrm{n}=53)$ & $(\mathrm{n}=41)$ \\
Serum LDH level $\mathrm{mg} \cdot \mathrm{dL}-1$ & $480 ; 1056$ & $431 \pm 533$ & $337 \pm 109$ \\
Serum CEA level $\mathrm{ng} \cdot \mathrm{mL}^{-1}$ & $(\mathrm{n}=129)$ & $(\mathrm{n}=40)$ \\
& $27.4 \pm 126.8$ & $17.8 \pm 59.8$ & $12.17 \pm 48.71$ \\
Serum TPA level U.L-1 & $(\mathrm{n}=126)$ & $(\mathrm{n}=48)$ & $(\mathrm{n}=40)$ \\
& $195.2 \pm 197.1$ & $159.7 \pm 126.1$ & $234.1 \pm 282.1$ \\
\end{tabular}

Results are presented as mean \pm SD. Numbers in parentheses represent the numbers of patients accessible for analysis, in the event of missing values. EL: elementary; MS: middle school; HS: high school; U: university. SDS: Self-rating Depression Scale; ECOG-PS: Eastern Co-operative Oncology Group - performance score; E: epidermoid carcinoma; A: adenocarcinoma; S: small cell anaplastic carcinoma; L: large cell anaplastic carcinoma; O: mixed or undefined subtype classification; T: tumour; N: node; M: metastasis; WBC: white blood cell; LDH: lactate dehydrogenase; CEA: carcino-embryonic antigen; TPA: tissue polypeptide antigen. No difference was found to be statistically significant, by means of the appropriate test (Pearson Chi-squared test, Student's t-test, Mann-Whitney U-test).

Table 2. - Self-rating Depression Scale (SDS) responses of the cohort studied

\begin{tabular}{|c|c|c|c|c|}
\hline SDS item & $\begin{array}{c}\text { None or } \\
\text { a little of } \\
\text { the time } \\
n\end{array}$ & $\begin{array}{l}\text { Some } \\
\text { of the } \\
\text { time } \\
n\end{array}$ & $\begin{array}{c}\text { Good } \\
\text { part of } \\
\text { the time } \\
n\end{array}$ & $\begin{array}{c}\text { Most or } \\
\text { all of } \\
\text { the time } \\
n\end{array}$ \\
\hline 1. I feel down-hearted, blue and sad & 65 & 49 & 3 & 16 \\
\hline 2. Morning is when I feel the best & 36 & 37 & 28 & 32 \\
\hline 3. I have crying spells or feel like it & 92 & 31 & 3 & 7 \\
\hline 4. I have trouble sleeping through the night & 55 & 37 & 19 & 22 \\
\hline 5. I eat as much as I used do & 47 & 41 & 14 & 31 \\
\hline 6. I enjoy looking at, talking to, and being with attractive women (or men) & 20 & 14 & 22 & 48 \\
\hline 7. I notice that I am losing weight & 54 & 29 & 14 & 35 \\
\hline 8. I have trouble with constipation & 90 & 25 & 6 & 12 \\
\hline 9. My heart beats faster than usual & 96 & 24 & 4 & 5 \\
\hline 10. I get tired for no reason & 49 & 49 & 16 & 17 \\
\hline 11. My mind is as clear as it used to be & 50 & 38 & 24 & 21 \\
\hline 12. I find it easy to do things I used to & 35 & 30 & 31 & 37 \\
\hline 13. I am restless and can't keep still & 73 & 37 & 10 & 13 \\
\hline 14. I feel hopeful about the future & 57 & 37 & 25 & 12 \\
\hline 15. I am more irritable then usual & 66 & 38 & 9 & 19 \\
\hline 16. I find it easy to make decisions & 42 & 41 & 28 & 21 \\
\hline 17. I feel that I am pretty useful and needed & 66 & 42 & 16 & 8 \\
\hline 18. My life is pretty full & 51 & 45 & 19 & 16 \\
\hline 19. I feel that others would be better off if I were dead & 114 & 8 & 3 & 6 \\
\hline 20. I still enjoy the things I used to do & 50 & 43 & 25 & 14 \\
\hline
\end{tabular}



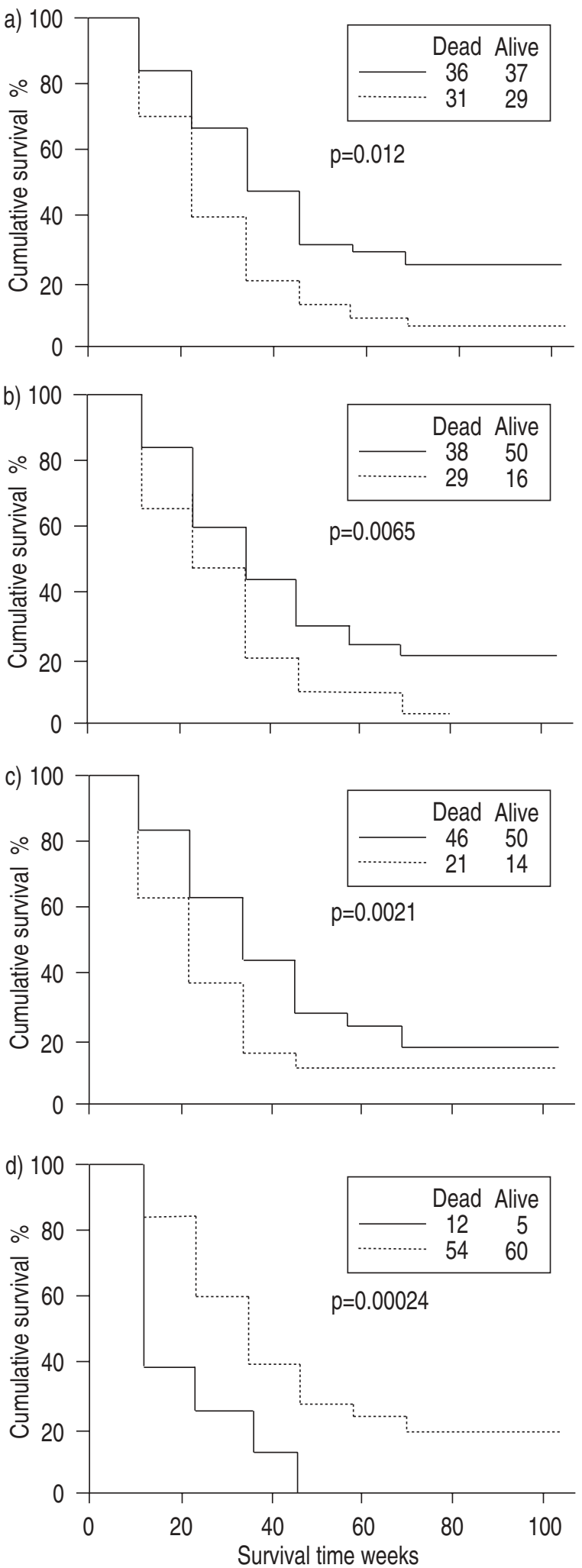

Fig. 1. - Survival probabilities based on the answers to four items of the Self-rated Depression Scale. Patients answers "always or often" $(-1$ ) or "never or rarely" ( ..........) in response to the following statements: a) item 2: "morning is when I feel the best"; b) item 5: "I eat as much as I used to"; c) item 18 "My life is pretty full"; d) item 19: "I feel that others would be better off if I were dead". Significance was determined using Gehan's generalized Wilcoxon test of the differences. The values in the boxes on each figure are the numbers of subjects dead or alive at the end of the study.
$8,12,15,18,19)$, being significant at $\mathrm{p}<0.0025$ in one case (item 19="I feel that others would be better off if I were dead"). Dichotomizing the sample into two groups with antithetical SDS responses (i.e. 1,2 versus 3,4 strata), item 18 emerged as a further significant factor (fig. 1). Fig. 2 shows the survival probability of the 95 patients who completed the entire questionnaire, according to their SDS index. As shown, depressed patients survived significantly less than the nondepressed subjects $(\mathrm{p}=0.048)$. After calculating the partial SDS indexes of the 38 incomplete questionnaires and analysing the survival of the entire cohort studied, results did not differ substantially $(\mathrm{p}=0.055)$.

Diverse multivariate analyses were performed to assess the prognostic value of mental depression in relation to the other prognostic determinants. First, the items of the Zung scale were considered individually. A broad model was designed, containing all the variables with possible prognostic significance (i.e. the eight previously mentioned SDS items, and the other 15 clinical and laboratory parameters listed in table 1). The analysis showed that SDS item 18, the need of help to fill out the questionnaire, and blood haemoglobin levels were, in order of decreasing importance, the most valuable factors among the 23 tested. Then, the analysis was restricted to the same SDS items and the three most important clinical variables (i.e. sex, the stage of disease and the ECOG-PS). A stepwise selection procedure was launched, which ended with the final model shown in table 3. SDS items 19,2, and 18, the stage of disease, and sex were all prognostically important, and, again, SDS items outweighed the clinical variables. Finally, a model was constructed using only the 20 items of the Zung scale: this model included 129 patients, had a global Chi-squared value of 29.78, and a p-value of 0.00023 .

To evaluate the overall significance of the depression instrument, a further model was tested containing age, sex, stage of disease, ECOG-PS, the need of help to fill out the questionnaire, the five laboratory parameters listed in table 1, and the SDS-index. Also in this type of analysis, a depressive status was extremely important, the most significant variable being the index developed by Zung $(\mathrm{p}=$ $0.0125)$.

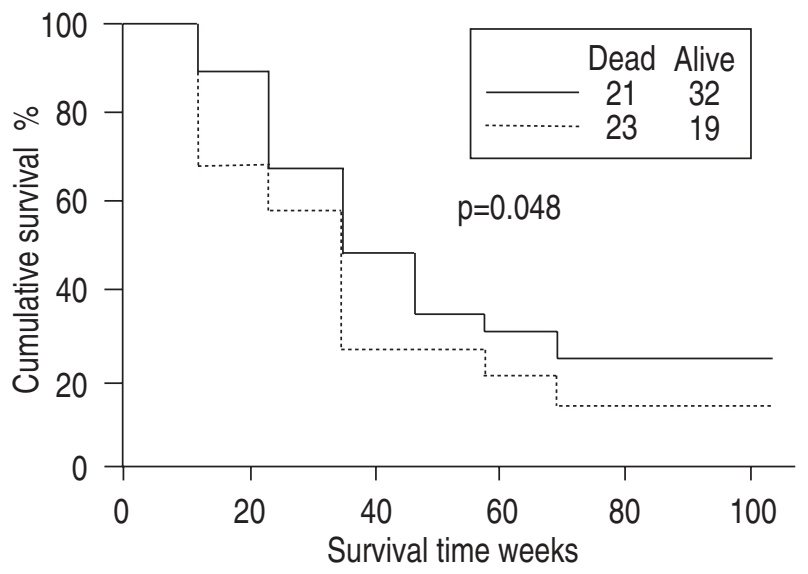

Fig. 2. - Survival probability of depressed (as determined by Self-Rating Depression Scale (SDS)-index $>50$ ) versus nondepressed patients (SDS index ð50). …....... SDS-index $>50$; — : SDS-index ð50. The difference was found to be significant according to Gehan's generalized Wilcoxon test. The values in the box are the number of subjects dead or alive at the end of the study. 
Table 3. - The best multivariate model for survival obtained

\begin{tabular}{lccc}
\hline Variable & RR & t-value & p-value \\
\hline $\begin{array}{l}\text { SDS item 198 (never or rarely=1, } \\
\text { often or always=2) }\end{array}$ & 4.6226 & 4.1561 & 0.0089 \\
$\begin{array}{l}\text { SDS item 2 (never or rarely=1, } \\
\text { often or always=2) }\end{array}$ & 2.4806 & 3.4073 & 0.0191 \\
$\begin{array}{l}\text { Stage of disease }(1,2,3 \mathrm{a}=3, \\
\quad 3 \mathrm{~b}=4,4=5)\end{array}$ & 1.3235 & 2.9586 & 0.0316 \\
$\begin{array}{l}\text { Sex (male=1, female=2) } \\
\text { SDS item 18 (never or rarely=1, }\end{array}$ & 0.2495 & -2.7714 & 0.0393 \\
$\quad$ often or always=2) & 1.5277 & 1.4921 & 0.1959 \\
\hline
\end{tabular}

Cox's proportional hazards regression analysis, stepwise backward selection, was used. Chi-squared $=44.83$, degrees of freedom $=5, \mathrm{p}<0.00001$. RR: risk ratio; SDS: self-rating depression scale.

\section{Discussion}

In the current study, the relationship between psychological depression and the prognosis of lung cancer was analysed. To the author's knowledge, this is the first prospective clinical evaluation of such a correlation. Findings are consistent and plausible. In brief, they show that mental depression is an important determinant of the patients' outcome. It could be even more important than any other prognostic factor, including the anatomical extent of the disease. The study comprises all patients seen for a new carcinoma of the lung in a single LC centre in little more than $1 \mathrm{yr}$. With the exception of a few cases with very advanced disease and low performance status, or patients showing severe intellectual impairment, the cohort studied reflects the LC population in the study province.

The design of the study had a few obvious limitations. For example, the low degree of patient's education. Most of the patients had elementary education, and experienced significant difficulty in understanding the questions on the SDS-questionnaire. Furthermore, they did not appear particularly enthusiastic about the study in spite of their voluntary participation, and thus their answers may have been unreliable. Another limitation was the heterogeneity of the population in relation to the clinical status of disease. Obviously, patients presented with different tumour stages ranging from small diseases undergoing evaluation for effective surgery to metastatic diseases planned for intensive schedules of chemoradiotherapy. Patients in this latter condition may have a particularly bad reaction to their disease and may be more susceptible to depression than surgically curable subjects. However it was verified that the presence of depression was poorly explained by the severity of the illness, as substantiated by the similar frequency of depression within different stages of disease.

Depression is a ubiquitous and universal human condition that extends on a continuum from normal mood swings to a pathological state [18]. The term "depression" may be used to describe different things. Depression can be an affect which is a subjective feeling tone of short duration; or a mood which is a state sustained over a longer period; or an emotion which comprises the feeling tones along with objective indications; or a disorder which has characteristic symptom clusters complexes and configurations [18]. Whether an effect, a symptom, or a disorder, the scales and inventories available for depression are numerous. Among them the scale described over $30 \mathrm{yrs}$ ago by ZuNG [15] retains many useful characteristics. It is all inclusive with respect to symptoms of the mental disorder; it is short and simple; it is quantitative rather than qualitative; it is self-administered and shows the patient's own response at the time the scale is taken. Furthermore the calculation of the derived index is an easy and rapid way to distinguish between depressed and nondepressed subjects. Another point in favour of the Zung scale is its availability in different languages and its validity documented in a cross-cultural survey [7].

Recognizing prognostic factors has several scopes in medicine. Prognostic factors may be helpful in advising individuals selecting treatment (when therapeutic options depend on the baseline clinical characteristics of the subject), adjusting for nonuniformity (when series from different locations and studies have to be considered) defining eligibility criteria and stratifying patients by risk subgroups and understanding (certain factors may provide insights into the disease process and provide direction for further studies). In LC, a small number of clinical characteristics are normally used to predict the outcome of patients [1, $26,27]$. Potentially useful prognostic factors however are much more numerous [28]. A partial list of such factors includes conventional biochemical tests [29-31], and newer tests based on the experimental evidence arising at the cellular and molecular level [32]. Preliminary reports suggest that additional factors might be important, all gathered under the comprehensive term of "quality of life" $[2,4,5]$. In 1995, a study dealing with QOL and prognosis was published by Buccheri et al. [16]. The study comprised 128 patients with LC, assessed for their QOL either before treatment or during the course of disease. That report showed a statistically significant correlation between patient survival and multiple aspects of QOL (in particular, the perception of limitations in physical autonomy, the feeling of insecurity, and a difficulty in having fun or relaxing). Some QOL scores remained significant after correcting for other prognostic factors, but it was not clear whether psychological factors were prognostically determinants by themselves, or, whether more simply, they were influenced by the physical status, the real indicator of prognosis. Furthermore, it was not possible to say which psychological symptom, if any, might be implicated.

With the current study, some of the above issues have been clarified. There is now sufficient evidence to say that psychological factors, and in particular the way in which patients face their disease (i.e. with a gloomy or an optimistic attitude) is prognostically very important. Of course, further studies are needed to support the evidence, and to evaluate the role of depression in more specific (and homogeneous) subgroup of patients.

\footnotetext{
Acknowledgements: The author would like to acknowledge the help of D. Ferrigno, and the dedication of M. Viale and L. Marino, nurses of the outpatients' unit of the A. Carle Hospital. He is also indebted to M. Tamburini, and C. Brunelli, for their helpful comments.
}

\section{References}

1. Minna JD, Higgins GA, Glatstein EJ. Cancer of the lung. In: De Vita V Jr, Hellman S, Rosemberg SA, eds. Cancer. 
Principles and Practice of Oncology. Philadelphia, J.B. Lippincott Co., 1985: pp. 507-597.

2. Kaasa S, Mastekaasa A, Lund E. Prognostic factors for patients with inoperable non-small cell lung cancer, limited disease. The importance of patients' subjective experience of disease and psychosocial well-being. Radiother Oncol 1989; 15: 235-242.

3. Buccheri G, Vola F, Ferrigno D. Aspects of quality of life in patients with lung cancer: a three observer evaluation study. Int J Oncol 1993; 2: 537-544.

4. Ganz PA, Lee JJ, Siau J. Quality of life assessment. An independent prognostic variable for survival in lung cancer. Cancer 1991; 67: 3131-3135.

5. Ruckdeschel JC, Piantadosi S, the Lung Cancer Study Group. Quality of life assessment in lung surgery for bronchogenic carcinoma. J Theor Surgery 1991; 6: 201205 (Abstract).

6. Buccheri G: The patient's perception of his own quality of life affects the prognosis of lung cancer. Supp Care Oncol 1995; 16: 11-13.

7. Bergman B, Aaronson NK, Ahmedzai S, Kaasa S, Sullivan M. The EORTC QLQ-LC13: a modular supplement to the EORTC core quality of life questionnaire (QLQC30) for use in lung cancer clinical trials. Eur J Cancer [A] 1994; 30A: 635-642.

8. Hollen PJ, Gralla RJ, Kris MG, Potanovich LM. Quality of life assessment in individuals with lung cancer: testing the Lung Cancer Symptom Scale (LCSS). Eur J Cancer [A] 1993; 29A Suppl. 1: S51-S58.

9. Tamburini M, Rosso S, Gamba A, Mencaglia E, De Conno F, Ventafridda V. A therapy impact questionnaire for quality-of-life assessment in advanced cancer research. Ann Oncol 1992; 3: 565-570.

10. Hopwood P. Progress, problems and priorities in quality of life research. Eur J Cancer [A] 1992; 28A: 17481752.

11. Zubrod CG, Scheiderman MA, Frei E, et al. Appraisal of methods for the study of chemotherapy in man: comparative therapeutic trial of nitrogen mustard and triethylene thiophosphoramide. J Chron Dis 1960; 11: 7-33.

12. Buccheri G, Biggi A, Ferrigno D, et al. Anti-CEA immunoscintigraphy and computed tomographic scanning in the preoperative evaluation of mediastinal lymph nodes in lung cancer. Thorax 1996; 51: 359-363.

13. World Health Organization. International Histological Classification of Tumours. Berlin, Springer-Verlag, 1991.

14. UICC. TNM Classification of Malignant tumours. Berlin, Springer-Verlag, 1987; p. 69.

15. Zung WWK. A self-rating depression scale. Arch Gen Psychiatry 1965; 12: 63-70.

16. Buccheri G, Ferrigno D, Tamburini M, Brunelli C. The patient's perception of his own quality of life might have an adjunctive prognostic significance in lung cancer. Lung Cancer 1995; 12: 45-58.

17. Zung WWK. A cross-cultural survey of symptoms in depression. Am J Psychiatry 1969; 126: 154-159.

18. Zung WWK. From art to science. Arch Gen Psychiatry 1973; 29: 328-337.

19. Siegel S. Nonparametric statistics for the Behavioural Sciences, New York, MacGraw Hill, 1956.

20. Kaplan EL, Meier F. Non-parametric estimation from incomplete observations. J Am Stat Assoc 1958; 58: 457481.

21. Lee ET, Desu MM. A computer program for comparing $\mathrm{K}$ samples with right-censored data. Computer Progr Biomed 1972; 2: 315-321.

22. Gehan EA. A generalized Wilcoxon test for comparing arbitrarily singly-censored samples. Biometrika $1965 ; 52$ : 203-223.

23. Cox DR. Regression models and life tables. J R Stat Soc 1972; 34: 187-220.

24. Buccheri G, Barberis P, Delfino MS. Diagnostic, morphologic, and histopathologic correlates in bronchogenic carcinoma. A review of 1045 bronchoscopic examinations. Chest 1991; 99: 809-814.

25. Buccheri G, Ferrigno D. Prognostic value of the tissue polyptide antigen in lung cancer. Chest 1992; 101: 12871292.

26. Stahel RA, Ginsberg R, Havemann K, et al. Staging and prognostic factors in small cell lung cancer: a consensus report. Lung Cancer 1989; 5: 119-126.

27. Feld R, Arriagada R, Ball DL, Mattson K, Sorensen JB. Prognostic factors in non-small cell lung cancer: a consensus report. Lung Cancer 1991; 7: 3-5.

28. Buccheri G, Ferrigno D. Prognostic factors in lung cancer: tables and comments. Eur Respir J 1994; 7: 13501364.

29. Kibbellaar RE, Moolenaar KEC, Michalides RJAM, et al. Neural cell adhesion molecule expression, neuroendocrine differentiation and prognosis in lung carcinoma. Eur J Cancer 1991; 27: 431-435.

30. Van der Gaast A, Van Putten WL, Oosterom R, Cozijnsen M, Hoekstra R, Splinter TAW. Prognostic value of serum thymidine kinase, tissue polypetide antigen and neuron specific enolase in patients with small cell lung cancer. $\mathrm{Br}$ J Cancer 1991; 64: 369-372.

31. Buccheri G, Ferrigno D, Vola F. Carcinoembryonic antigen (CEA), tissue polyptide antigen (TPA), and other prognostic indicators in the squamous cell carcinoma of the lung. Lung Cancer 1993; 10: 21-33.

32. Pedersen AC, Bach FW, Nissen M, Bach F. Creatine kinase BB and beta-2-microglohulin as markers of CNS metastasis in patients with small-cell lung cancer. J Clin Oncol 1985; 3: 1364-1372. 